\title{
Demonstration of \\ Orbital-Angular-Momentum-Based Optical Switching Using Dual-Area Mirrors
}

\author{
Shengtao Chen ${ }^{*}$, Xizheng Ke ${ }^{1,2}$ \\ ${ }^{1}$ Xi'an University of Technology, School of Automation and Information Engineering, Xi'an, China \\ ${ }^{2}$ Shaanxi Civil-Military Integration Key Laboratory of Intelligence Collaborative Networks, Xi'an, China \\ Email: *rxjh1063697957@163.com
}

How to cite this paper: Chen, S.T. and Ke, X.Z. (2021) Demonstration of OrbitalAngular-Momentum-Based Optical Switching Using Dual-Area Mirrors. Optics and Photonics Journal, 11, 351-359. https://doi.org/10.4236/opj.2021.118025

Received: July 10, 2021

Accepted: August 7, 2021

Published: August 10, 2021

Copyright $\odot 2021$ by author(s) and Scientific Research Publishing Inc. This work is licensed under the Creative Commons Attribution International License (CC BY 4.0).

http://creativecommons.org/licenses/by/4.0/ (c) (i) Open Access

\begin{abstract}
We have designed an optical separation device called dual-area mirror for the data link of orbital angular momentum (OAM) multiplexing. Experiments show that the OAM multiplexed beams can be switched by using dual-area mirrors, using OOK to modulate four channels separately to form two inputs, channel A and channel B. There are two OAM beams that are multiplexed in each of channel A and channel B, using a spatial light modulator (SLM) to convert the OAM multiplexed beams in each channel. One of the beams is converted into a Gaussian beam, and then separated by a dual-area mirror, so as to realize the switch of a beam carrying different data in the two channels. Then these channels are detected. The waveform indicates that the switch is successful, and the measured optical power indicates that the dual-area mirror can reduce the bit error rate in the communication links. In addition, the device reduces the experimental cost, is easy to implement, is easy to integrate, and increases the angle between the separated beams.
\end{abstract}

\section{Keywords}

Optical Communication, Dual-Area Mirror, Switching, Optical Network

\section{Introduction}

Multiplexing multiple data channels as a method to increase system capacity has been widely used in optical networks [1] [2]. In recent years, a new method has emerged, that is, the use of orbital angular momentum (OAM) beams to carry data information for multiplexing [3] [4] [5] [6]. Because the OAM beams with different topological charges $(I)$ are orthogonal to each other [7], the specific 
modes of OAM can be used as the routing and switching identifiers in optical networks [8] [9] [10]. In a mature communication system, an important index to improve the flexibility and practicability of the system includes flexible conversion and manipulation between channels. At present, the research focus of OAM optical communication includes this routing technology for end-to-end signal transmission path conversion [11] [12]. When different data streams are modulated to different OAM modes in the OAM multiplexing system, switching the data information carried between the OAM modes can increase the flexibility of data processing and management in communication links [13]. Generally, OAM multiplexing communication systems in free space are mostly static and pointto-point links. However, the application of OAM to achieve reconfigurable network functions in a multiuser environment has more advantages. Therefore, it is an important development trend in the future to selectively manipulate different OAM data channels and realize OAM-based reconfigurable optical networks [14] [15] [16] [17].

Common methods for separating the coaxially transmitted OAM beam from Gaussian beam, for example, 1) Using a beam splitter (BS) and pinholes to separate [18], this method requires many experimental devices, the construction of the optical path occupies a large area, is not easy to integrate, and the BS loses a lot of optical power; 2) Using a SLM to load a specially designed grating for separation [19], this method is difficult to experiment, the separation angle between the OAM beam and the Gaussian beam is small, the cost is high, and the optical power loss of SLM is also large. In this paper, we propose a new method of beam separation, which also realizes the selective combination of OAM beams from input to output. We demonstrate experimentally the reconfigurable optical switching based on OAM. Using the reflective spatial light modulators (SLMs) and the designed dual-area mirrors [20], the OAM multiplexed beams are converted, spatially separated, recombined, restored to the initial state, and finally sent to the output.

\section{Concept and Principle}

Figure 1(A) shows the dual-area mirror. The inner area and the outer area are two independent areas, and any angle difference can be formed between them. In the experiment of this paper, we set the inner area radius to $\mathrm{r}=1.5 \mathrm{~mm}$ and the outer area radius to $\mathrm{R}=15 \mathrm{~mm}$ according to the Gaussian spot radius of 0.9 $\mathrm{mm}$. Figure 1(B) shows the separation of a Gaussian beam and an OAM beam transmitted coaxially. The intensity distribution of the OAM beam is a donut shape with zero intensity at the center [10]. When different OAM modes are multiplexed, they will be in the same position in space [20], and the Gaussian beam will always be located in the center of the OAM beam when it is transmitted coaxially with the OAM beam. When the coaxially transmitted OAM beam and Gaussian beam are emitted to the dual-area mirror, the Gaussian beam will be emitted to the central area and the OAM beam will be emitted to the outer 


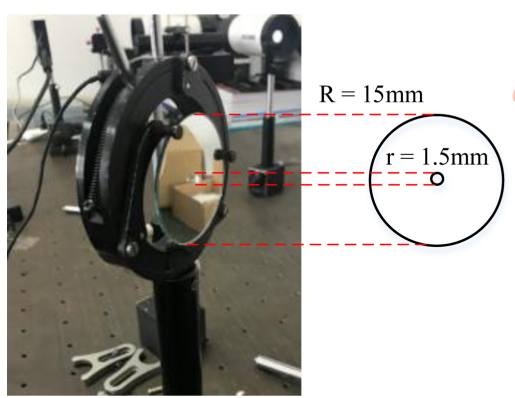

(A)

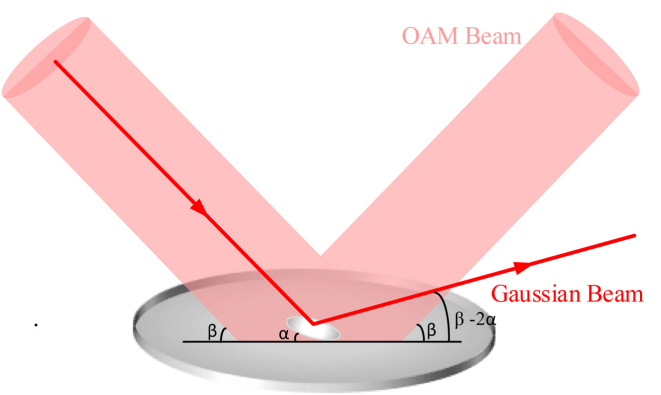

(B)

Figure 1. (A) Dual-area mirror and (B) Principle of separating multiplexed beams.

area. Since the exit angle of the light beam is equal to the incident angle when the beam is reflected, the separation can be completed by adjusting the angle between the inner area and the outer area of the dual-area mirror.

Figure 2 shows the principle of OAM-based optical switching. The different size of each ring in the two channels indicates that the topological charge of each OAM beam is different, and the different color of each ring indicates that the data carried by each OAM beam is different. Load the corresponding phase diagram to the OAM multiplexed beams in channel A and convert one beam to be switched into a Gaussian beam, the other beam still carries the OAM state because the topological charge is not converted to 0 . Finally, the Gaussian beam is separated from the OAM beam. Do the same operation to channel B as channel A.

The Gaussian beam separated from channel $A$ is introduced into the OAM beam of channel B, and the Gaussian beam separated from channel B is introduced into the OAM beam of channel A. The recombined beams are transmitted coaxially, and the multiplexed beams in channel A and channel B are converted back to the initial topological charge states by loading the corresponding phase diagrams. By comparing before and after switch, all OAM states have not changed. We switched the data carried by the beam $l_{3}$ in channel A and channel $\mathrm{B}$.

This type of optical switching experiment has no theoretical limit on the number of channels and the number of OAM beams multiplexed in each channel. And the more the number of channels and the multiplexing number of OAM beams in each channel. The more options for switching, the more flexible the entire system, and the easier it is to build a large-scale OAM-based optical network. As shown in Figure 3.

\section{Experiment}

Figure 4 shows the optical path of the OAM-based optical switching experiment. All light sources are He-Ne lasers with a wavelength of $632.8 \mathrm{~nm}$, and all data sources are generated by signal generators. The data source 1 in channel A is sent to SLM1 and converted into an OAM beam with $l_{1}=+2$. The light intensity is shown as (a) in Figure 4, and (a1) is the interferogram of (a). Data source 2 is sent to SLM2 and converted into an OAM beam with $l_{2}=+6$, the light intensity is as shown in (b). Use BS1 to combine beams to form OAM multiplexing, 


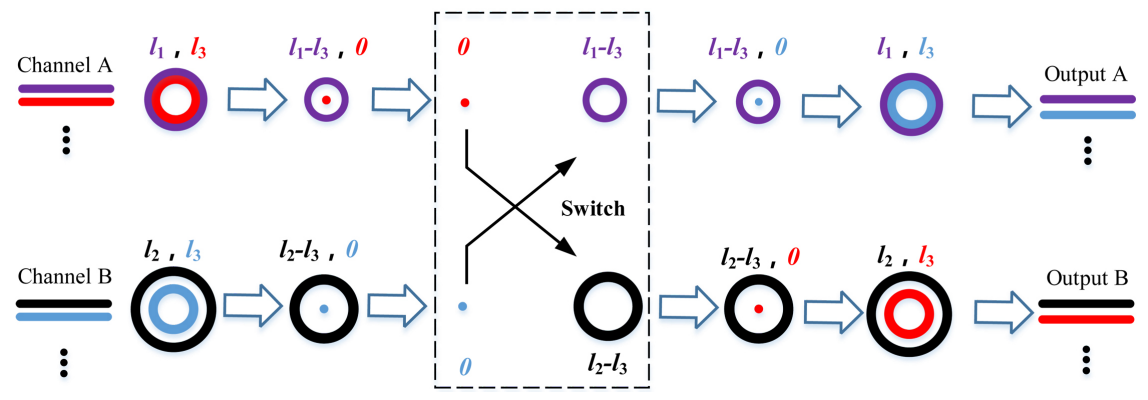

Figure 2. Principle of OAM-based optical switching.
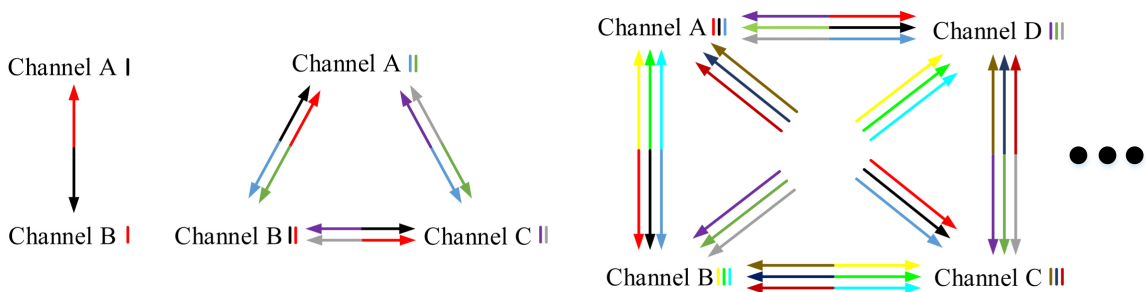

Figure 3. OAM-based networks constructed under different number of channels and different number of OAM multiplexed beams.

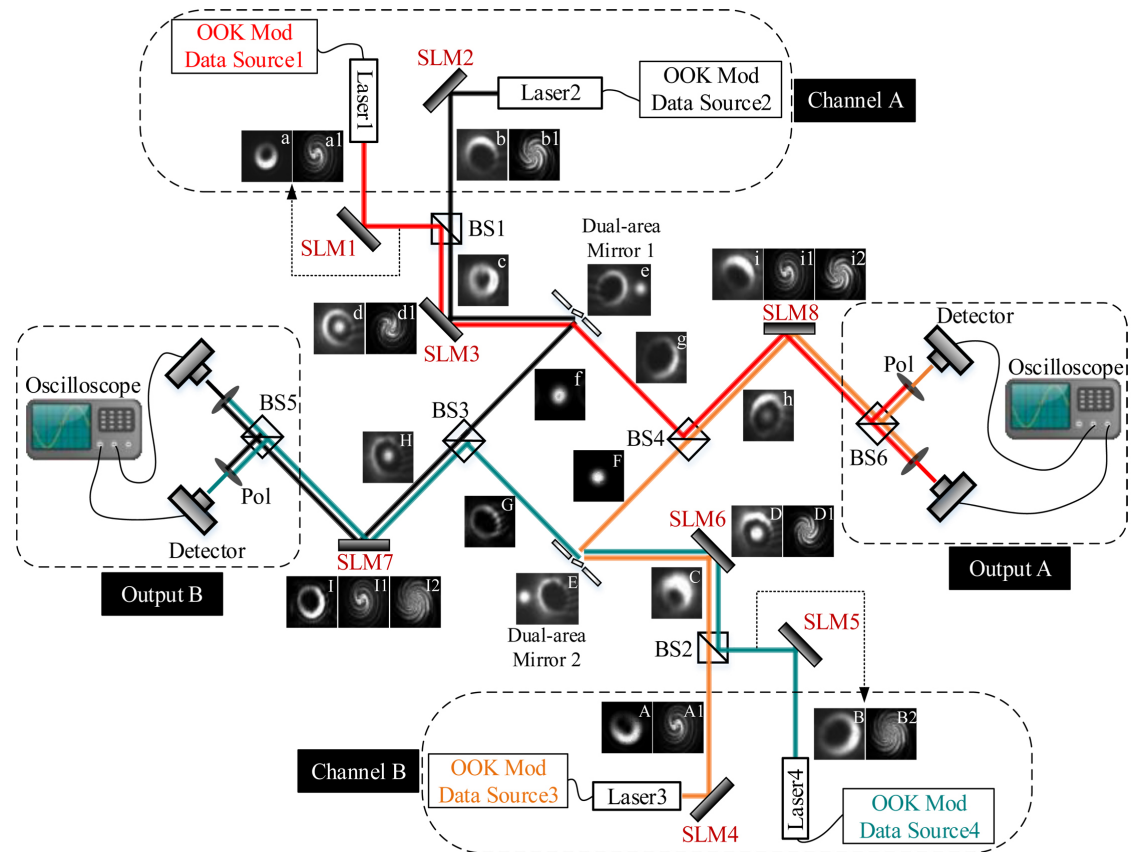

Figure 4. Experiment setup (Pol, polarizer).

(c) is the light intensity of the OAM multiplexed beams. Use SLM3 to convert $l_{1}$ into a Gaussian beam, while $I_{2}$ is still an OAM beam, and they are transmitted coaxially as shown in (d). The topological charge of $l_{2}$ has also been converted, as shown in (d1). The dual-area mirror 1 separates the OAM beam from the Gaussian beam, as shown in (e). Channel B and channel A are more like mirror manipulation. The difference is that the topological charges of the two OAM multiplexed beams in channel $\mathrm{B}$ are $l_{3}=+2$ and $l_{4}=+8$, and they carry different data 
sources.

Recombine the Gaussian beam (f) separated in channel A with the OAM beam $(G)$ separated in channel $B$, as shown in $(H)$. Recombine the Gaussian beam $(F)$ separated in channel B with the OAM beam ( $g$ ) separated in channel A, as shown in (h). Use SLM7 and SLM8 to restore them to the initial OAM states. Since the OAM beams with different topological charges are orthogonal when they are superimposed, we use the polarizers to filter the multiplexed beams, and connect the detectors to the oscilloscopes for detection.

\section{Experimental Result}

Figure 5 shows the signal waveforms of channel A and channel B collected before and after optical switching. We distinguish four signals by waveforms of different frequencies. Different OAM beams and their corresponding signal waveforms are identified by the same color. In channel $\mathrm{A}$, beam $l_{1}=+2$ is multiplexed with beam $l_{2}=+6$, and in channel $\mathrm{B}$, beam $l_{3}=+2$ is multiplexed with beam $l_{4}=+8$. The data they carry are displayed by multichannel oscilloscopes. In output $\mathrm{A}$, we can see that the beam $l_{2}=+6$ in channel $\mathrm{A}$ is recombined with the beam $l_{3}=+2$ in channel $B$. In output $B$, we can see that the beam $l_{4}=+8$ in channel $B$ is recombined with the beam $l_{1}=+2$ in channel $A$. It shows that the data carried by the OAM beams with the topological charge of +2 in channel $\mathrm{A}$

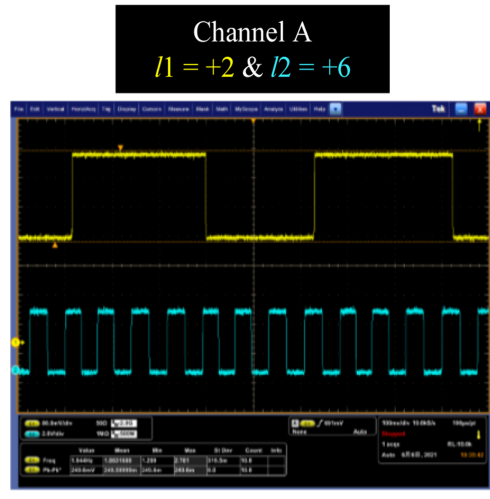

Before the switch

\section{Channel B}

$13=+2 \& l 4=+8$

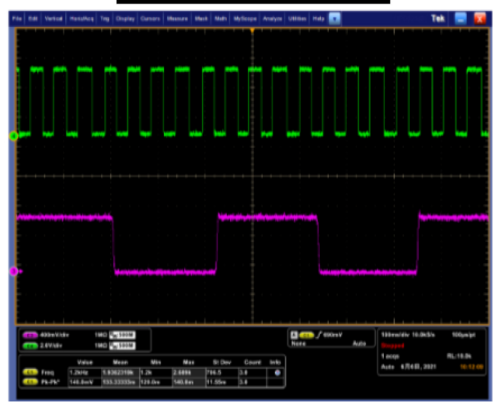

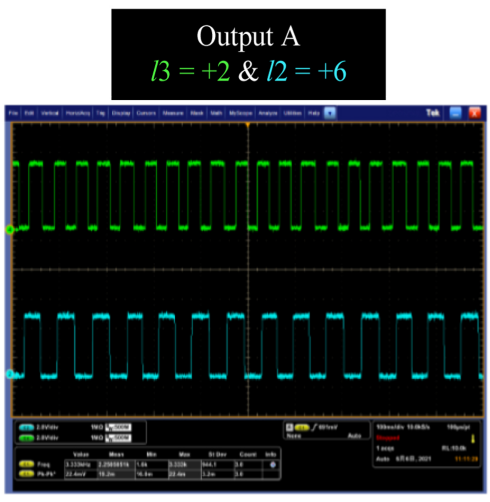

After the switch

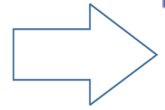

Output B $l 1=+2 \& l 4=+8$

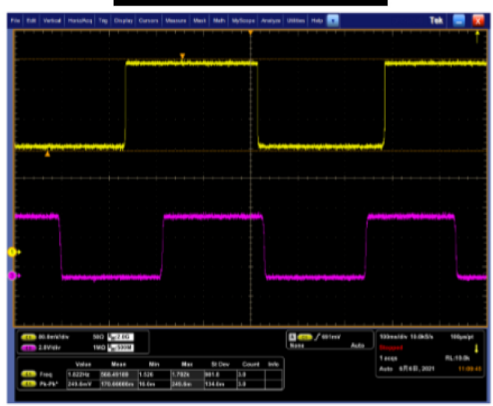

Figure 5. Waveforms collected before and after switch. 


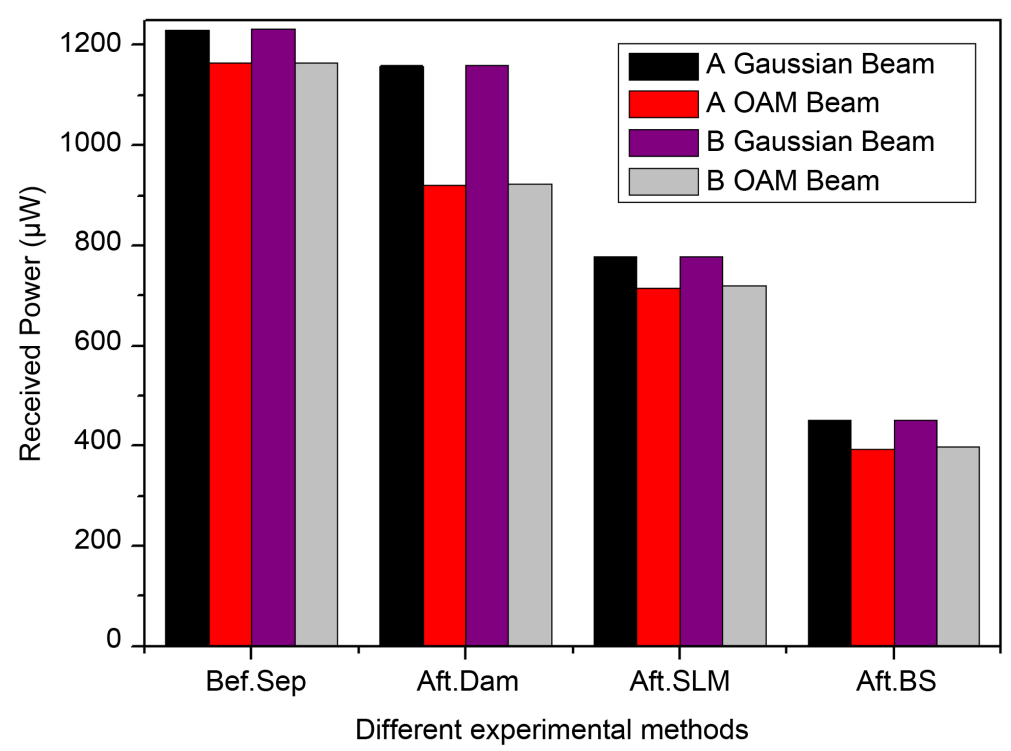

Figure 6. Received optical power under different methods. (Bef, before; Aft, after; Sep, separation; Dam, dual-area mirror).

and channel B are successfully switched, and compared with the output and input, the OAM state has not changed.

We use the two separation methods mentioned in Section 1 and the method proposed in this paper to carry out the experiments in turn, and the collected received optical power is shown in Figure 6. The experiment measured that the average power of the Gaussian/OAM beam in channel A before separation is $1230.6 \mu \mathrm{W} / 1163.8 \mu \mathrm{W}$, the average power after separation using dual-area mirror is $1159 \mu \mathrm{W} / 921.2 \mu \mathrm{W}$, the average power after separation using SLM is 777.2 $\mu \mathrm{W} / 717.4 \mu \mathrm{W}$, and the average power after separation using BS and pinholes is $451.6 \mu \mathrm{W} / 394.4 \mu \mathrm{W}$.

The average power of the Gaussian/OAM beam in channel B before separation is $1232.4 \mu \mathrm{W} / 1165.2 \mu \mathrm{W}$, the average power after separation using dual-area mirror is $1160.2 \mu \mathrm{W} / 923 \mu \mathrm{W}$, the average power after separation using SLM is $778.6 \mu \mathrm{W} / 719.8 \mu \mathrm{W}$, and the average power after separation using BS and pinholes is $452.4 \mu \mathrm{W} / 397.8 \mu \mathrm{W}$. It can be seen that the experimental effect of using the dual-area mirror is the best, and the loss of optical power is the minimal. In reconfigurable data switch, with the decrease of optical power loss, the bit error rate will decrease [3]. Therefore, the dual-area mirror can improve the transmission quality of the optical communication links.

\section{Discussion}

We propose a method to separate the coaxially transmitted Gaussian beam from OAM beam using a dual-area mirror, and use this method to realize an OAMbased optical switch experiment. The results show that the device we designed can greatly reduce the optical power loss, thereby reducing the bit error rate of the communication links. And the method greatly reduces the experimental cost. 
If SLMs are used in the beam separation part to replace the dual-area mirrors, then we need 10 SLMs and 6 BSs to complete the experiment in Figure 4. If we use beam splitters and pinholes instead of dual-area mirrors, we need 8 SLMs, 8 BSs and 4 pinholes to complete the experiment in Figure 4. Our method only needs 8 SLMs, 6 BSs and 2 dual-area mirrors to complete the experiment. As we all know, the price of mirrors is cheap. At the same time, it also has many advantages. For example, it is easier to integrate, can separate beams at a large angle, and can be applied to a variety of OAM reconstruction scenes. Therefore, the research results of this paper contribute to the construction of large-scale and flexible OAM-based optical networks.

\section{Acknowledgements}

This work was funded by the Key Industry Innovation Chain Project of Shaanxi Province (Grant No. 2017ZDCXL-GY-06-01), the Scientific Research Program of Education Department of Shaanxi Province (Grant No. 18JK0341), and Xi'an Science and Technology Innovation Guidance Project [Grant No. 201805030YD8CG14(12)].

\section{Conflicts of Interest}

The authors declare no conflicts of interest regarding the publication of this paper.

\section{References}

[1] Ramaswami, R., Sivarajan, K. and Sasaki, G. (2010) Optical Networks: A Practical Perspective. Morgan Kaufmann Publishers, Boston.

https://www.researchgate.net/publication/216545893 Optical Networks A Practic al Perspective

[2] Mokhtar, A. and Azizoğlu, M. (1998) Adaptive Wavelength Routing in All-Optical Networks. IEEE/ ACM Transactions on Networking, 6, 197-206. https://doi.org/10.1109/90.664268

[3] Wang, J., Yang, J.Y., Fazal, I.M., Ahmed, N., Yan, Y., Huang, H., Ren, Y.X., Yue, Y., Dolinar, S., Tur, M. and Willner, A.E. (2012) Terabit Free-Space Data Transmission Employing Orbital Angular Momentum Multiplexing. Nature Photonics, 6, 488-496. https://doi.org/10.1038/nphoton.2012.138

[4] Fazal, I.M., Ahmed, N., Wang, J., Yang, J.Y., Yan, Y., Shamee, B., Huang, H., Yue, Y., Dolinar, S., Tur, M. and Willner, A.E. (2012) 2 Tbit/s Free-Space Data Transmission on Two Orthogonal Orbital-Angular-Momentum Beams Each Carrying 25 WDM Channels. Optics Letters, 37, 4753-4755.

https://doi.org/10.1364/OL.37.004753

[5] Huang, H. Xie, G.D., Yan, Y., Ahmed, N, Ren, Y.X., Yue, Y., Rogawski, D., Tur, M., Erkmen, B., Birnbaum, K., Dolinar, S., Lavery, M., Padgett, M.J. and Willner, A.E. (2013) $100 \mathrm{Tbit} / \mathrm{s}$ Free-Space Data Link Using Orbital Angular Momentum Mode Division Multiplexing Combined with Wavelength Division Multiplexing. Optical Fiber Communication Conference, Anaheim, 17-21 March 2013, OTh4G.5. https://doi.org/10.1364/OFC.2013.OTh4G.5

[6] Yue, Y., Bozinovic, N., Ren, Y.X., Huang, H., Tur, M., Kristensen, P., Ramachan- 
dran, S. and Willner, A.E. (2013) 1.6-Tbit/s Muxing, Transmission and Demuxing through 1.1-km of Vortex Fiber Carrying 2 OAM Beams Each with 10 Wavelength Channels. Optical Fiber Communication Conference, Anaheim, 17-21 March 2013, OTh4G.2. https://doi.org/10.1364/OFC.2013.OTh4G.2

[7] Djordjevic, I.B. (2011) Deep-Space and Near-Earth Optical Communications by Coded Orbital Angular Momentum (OAM) Modulation. Optics Express, 19, 1427714289. https://doi.org/10.1364/OE.19.014277

[8] Yao, A.M. and Padgett, J.M. (2011) Orbital Angular Momentum: Origins, Behavior and Applications. Advances in Optics and Photonics, 3, 161-204. https://doi.org/10.1364/AOP.3.000161

[9] Bozinovic, N., Yue, Y., Ren, Y., Tur, M., Kristensen, P., Huang, H., Willner, A.E. and Ramachandran, S. (2013) Terabit-Scale Orbital Angular Momentum Mode Division Multiplexing in Fibers. Science, 340, 1545-1548.

https://doi.org/10.1126/science.1237861

[10] Allen, L., Beijersbergen, M.W., Spreeuw, R.J.C. and Woerdman, J.P. (1992) Orbital Angular Momentum of Light and the Transformation of Laguerre-Gaussian Laser Modes. Physical Review, 45, 8185-8189. https://doi.org/10.1103/PhysRevA.45.8185

[11] Tomkos, I., Azodolmolky, S., Sole-Pareta, J., Careglio, D. and Palkopoulou, E. (2014) A Tutorial on the Flexible Optical Networking Paradigm: State of the Art, Trends, and Research Challenges. Proceedings of the IEEE, 102, 1317-1337. https://doi.org/10.1109/JPROC.2014.2324652

[12] Mamadou, D., Shen, F., Dedo, M., Zhou, Q.F., Guo, K. and Guo, Z.Y. (2019) High-Efficiency Sorting and Measurement of Orbital Angular Momentum Modes Based on the March-Zehnder Interferometer and Complex Phase Gratings. Measurement Science and Technology, 30, Article ID: 075201.

https://iopscience.iop.org/article/10.1088/1361-6501/ab0e62 https://doi.org/10.1088/1361-6501/ab0e62

[13] Wang, J., Yang, J.Y., Fazal, I.M., Ahmed, N., Yan, Y., Willner, A.E., Dolinar, S. and Tur, M. (2012) Experimental Demonstration of 100-Gbit/s DQPSK Data Exchange between Orbital-Angular-Momentum Modes. Optical Fiber Communication Conference, Los Angeles, 4-8 March 2012, OW1I.5. https://doi.org/10.1364/OFC.2012.OW1I.5

[14] Ahmed, N., Huang, H., Ren, Y.X., Yan, Y., Xie, G.D., Tur, M. and Willner, A.E. (2014) Reconfigurable $2 \times 2$ Orbital Angular Momentum Based Optical Switching of 50-Gbaud QPSK Channels. Optics Express, 22, 756-761. https://doi.org/10.1364/OE.22.000756

[15] Willner, A.E., Li, L., Xie, G.D., Ren, Y.X., Huang, H., Yue, Y., Ahmed, N., Willner, M.J., Willner, A.J., Yan, Ya., Zhao, Z., Wang, Z., Liu, C., Tur, M. and Ashrafi, S. (2016) Orbital-Angular-Momentum-Based Reconfigurable Optical Switching and Routing. Photonics Research, 4, 5-8. https://doi.org/10.1364/PRJ.4.0000B5

[16] Yue, Y., Huang, H., Ahmed, N., Yan, Y., Ren, Y.X., Xie, G.D., Rogawski, D., Tur, M. and Willner, A.E. (2013) Reconfigurable Switching of Orbital-Angular-MomentumBased Free-Space Data Channels. Optics Letters, 38, 5118-5121. https://doi.org/10.1364/OL.38.005118

[17] Willner, A.E., Huang, H., Ahmed, N., Yue, Y. and Willner, M.J. (2014) Data Switching in Communication Networks Using Orbital-Angular-Momentum Multiplexing. Photonics in Switching 2014, San Diego, 13-17 July 2014, PT1B.1. https://doi.org/10.1364/PS.2014.PT1B.1

[18] Ahmed, A., Huang, H., Yue, Y., Yan, Y., Ren, Y.X. and Willner, A.E. (2012) Dem- 
onstration of Add/Drop Multiplexer for 100-Gbit/s RZ-QPSK Channels over Spatially Multiplexed Orbital Angular Momentum Modes. IEEE Photonics Conference, Burlingame, 23-27 September 2012, 600-601.

https://doi.org/10.1109/IPCon.2012.6358764

[19] Huang, H., Yue, Y., Yan, Y., Ahmed, N., Ren, Y.X., Tur, M. and Willner, A.E. (2013) Liquid-Crystal-on-Silicon-Based Optical Add/Drop Multiplexer for Orbital-Angular-Momentum-Multiplexed Optical Links. Optics Letters, 38, 5142-5145. https://doi.org/10.1364/OL.38.005142

[20] Ke, X.Z. and Chen, S.T. (2020) Reconfigurable Orbital Angular Momentum Exchange Assisted by Dual-Area Mirror. Optics Engineering, 59, 116104-116111. https://doi.org/10.1117/1.OE.59.11.116104 